\title{
„Unter der Maske der Liebe“ - Häusliche Gewalt in Last Friends
}

\section{Fauve Görlach}

\section{$1 \quad$ Einleitung}

Es gibt ein Zitat von Leonardo da Vinci: „Wo die Liebe beginnt, hört die Gewalt auf. Liebe siegt über alles." (zit. nach Meyer 2009: 23). Genau umgekehrt verhält es sich bei der häusliche Gewalt - man könnte sagen, wo die Liebe beginnt, fängt auch die Gewalt an. Dieser Aufsatz macht es sich zur Aufgabe, das Thema häuslicher Gewalt in Japan zu beleuchten und aufzuzeigen, wie dieser Problemkreis in der Fernsehserie (terebi dorama) Last Friends verarbeitet wird.

Last Friends ist eine elfteilige Serie über Freundschaft, Liebe, häusliche Gewalt (domestic violence), soziale Erwartungen und auch Homo- bzw. Transsexualität. Fünf junge Menschen leben in einer Wohngemeinschaft (von ihnen Share House genannt) zusammen und werden dabei mit ganz unterschiedlichen Problemen konfrontiert. Michiru, die im Zentrum der Analyse dieses Aufsatzes steht, hat zuvor mit ihrem Freund Sōsuke zusammengewohnt, der sie seelisch und körperlich misshandelt hat. Für sie ist die WG ein Ort, an den sie sich geflüchtet hat. Die Mitbewohnerin Ruka hat sich in Michiru verliebt und kämpft um eine Anerkennung ihrer sexuellen Orientierung und damit auch ihrer Person. Gegen Vorurteile kämpft auch Takeru, der nicht dem gängigen Männlichkeitsideal entspricht. Weitere Mitbewohner sind Eri, die die wahre Liebe sucht und dabei ihre Ängste mit Frohsinn überspielt, und Ogurin, der auch auf der Suche nach der wahren Liebe ist, jedoch von seiner fremdgehenden Frau nicht loskommt. Am Ende der Serie findet jeder der fünf sein eigenes Glück und seinen inneren Frieden. Michiru gründet zusammen mit Ruka und Takeru eine kleine glückliche Familie, in der sie Sōsukes und ihr Kind behütet aufziehen kann.

Last Friends wurde vom 10. April bis zum 19. Juni 2008 donnerstags um 22 Uhr bei Fuji TV ausgestrahlt. Durchschnittlich haben 17,7\% der Haushalte in der Kantō-Region das dorama verfolgt. ${ }^{1}$ Die letzte Folge haben sogar 22,8\% gesehen.

1 Durchschnitt errechnet anhand der Daten von Video Research Ltd. 
Die Spitzenquote von 25,9\% wurde während der letzten Szene erreicht (vgl. Tokyograph 2008). Last Friends erhielt außerdem einige Auszeichnungen bei den 1st Tokyo Drama Awards, bei den 57th Television Drama Academy Awards und beim 12th Nikkan Sports Drama Grand Prix. Das spricht für die positive Resonanz der Zuschauer und Kritiker.

Dass das Thema der häuslichen Gewalt so direkt angesprochen wird und auf positive Reaktionen trifft ist nicht selbstverständlich. Bis in die 1980er Jahre wurde häusliche Gewalt in Japan als ein nicht vorhandenes Problem angesehen; erst durch den Einsatz von Frauengruppen entstand eine vermehrte Aufmerksamkeit und das Problem wurde anerkannt. Zunächst war das Phänomen auch begrifflich schwer zu fassen, da in Japan unter „häuslicher Gewalt“ eher die Gewalt von Kindern gegenüber ihren Eltern als die Gewalt zwischen Eheleuten oder einem Paar verstanden wurde. Als die Öffentlichkeit auf das Thema der Gewalt zwischen Eheleuten aufmerksam wurde, wurde deshalb nach einem Begriff für diese Art von häuslicher Gewalt gesucht, und man einigte sich schließlich auf domestic violence (abgekürzt $D V$ ), das aus dem Englischen übernommen wurde.

In dieser Arbeit wird analysiert, wie die häusliche Gewalt in Last Friends dargestellt wird und welche Ursachen dafür angegeben werden. Nach einer kurzen Vorstellung der einzelnen Charaktere wird anhand einzelner Szenen untersucht, wie Gewalt in der Serie thematisiert wird. Die Analyse wird zeigen, ob und inwieweit die Darstellung an die Wirklichkeit angelehnt ist und welche Hintergründe für die Handlungen angegeben werden. Dadurch soll auch klar werden, welches Bild dem Zuschauer vermittelt wird und welche Auswirkungen dies möglicherweise haben könnte.

Der zweite Teil der Arbeit widmet sich der aktuellen Situation in Japan, indem die Gesetzeslage erläutert wird und die Ergebnisse einer landesweiten Kabinettsumfrage diskutiert werden. Dieser Teil soll noch tiefere Einblicke in die Materie ermöglichen und die Lage der betroffenen Frauen verdeutlichen. So kann die Realitätsnähe der Darstellungsweise im dorama überprüft werden. 


\section{Die Charaktere}

In diesem Abschnitt werden die Charaktere vorgestellt, die für das Thema der häuslichen Gewalt in dem dorama Last Friends relevant sind. Das sind zum einen Aida Michiru ${ }^{2}$, die von der häuslichen Gewalt betroffene Frau, und zum anderen der Täter Oikawa Sōsuke.

\subsection{Aida Michiru}

Eine der drei Hauptfiguren des doramas Last Friends ist Aida Michiru, eine 22jährige Friseurassistentin. Sie kommt aus einer zerrütteten Familie, da sowohl ihr Vater als auch die Mutter Alkoholiker sind und der Vater die Familie verlassen hat, als sie in der Mittelstufe war. Ihre Mutter ist mit ihr aus Tōkyō weggezogen und hat sie allein großgezogen. Als sie nach einigen Jahren wieder nach Tōkyō zurückziehen, trifft Michiru ihre Freundin Kishimoto Ruka wieder.

Ihre Mutter verbringt viel Zeit mit anderen Männern. Ihrer Ansicht nach ist es normal, dass der Mann die Frau schlägt (Folge 9, 19:00). Dies ist ein Relikt aus den Zeiten des ie-Systems ${ }^{3}$, bei dem die Frau unter dem Mann stand und der Mann die Macht über die Frau hatte. Die Gedanken, die damit in Zusammenhang stehen, sind auch heute noch nicht gänzlich aus der japanischen Gesellschaft verschwunden: „In Japan, the idea of a male-dominated society is deeply rooted. There is still a tendency not to recognize the social status of women." (Watanabe 2002: 90). Genau deswegen hat die Serie einen aufklärenden Charakter: Sie spielt in der heutigen Zeit, behandelt die Probleme junger Menschen und macht sie so auch für die älteren Generationen verständlich.

Michiru wohnt zusammen mit ihrer Mutter, unterstützt sie finanziell und kümmert sich um den Haushalt. Sie hat einen Freund, Oikawa Sōsuke, und sie scheinen zu Beginn sehr glücklich miteinander zu sein. Als die Mutter, die sowieso kaum Interesse an ihrer Tochter zeigt, einen neuen Mann nach Hause mit-

2 Die Namen werden hier wie im Japanischen angegeben, d. h. zuerst wird der Nachname und dann der Vorname genannt.

3 Das ie-System (ie = Haus, Familie) war ein Ständesystem, das seit der Edo-Zeit (1603-1868) bis zum Ende des 2. Weltkrieges die Hierarchie in der Familie organisierte. So hatte das männliche Familienoberhaupt weitreichende Vollmachten über die Familienmitglieder, und Frauen waren sowohl ihren Vätern als auch Männern und Söhnen untergeordnet. 
bringt, fühlt sich Michiru verlassen. Die einzige Person, auf die sie sich stützt, ist ihr Freund. Er hat Ähnliches erlebt und sie glaubt, dass er sie verstehen kann. Als dieser ihr den Vorschlag macht, in eine gemeinsame Wohnung zu ziehen, scheint alles eine gute Entwicklung zu nehmen. Jedoch kann man im Verlauf der Serie beobachten, wie die selbstsichere und fröhliche Michiru mehr und mehr in Schwierigkeiten kommt und an der Situation zerbricht.

\subsection{Oikawa Sōsuke}

Der 23-jährige Oikawa Sōsuke arbeitet bei der Kinderfürsorge des Bezirksrathauses und kümmert sich um Fälle, in denen Kinder allein zurückgelassen wurden. Wie Michiru ist er in ungewöhnlichen und schwierigen Familienverhältnissen aufgewachsen: Seine Mutter, die ihn allein großgezogen hat, reichte ihn in der Familie herum, nachdem sie einen neuen Liebhaber gefunden hatte. Nach außen hin wirkt der junge Mann sehr nett und fürsorglich: So kümmert er sich im Verlauf der Serie zum Beispiel um einen kleinen Jungen und bringt sich für ihn in Gefahr. Aus diesem Grund ist die Entwicklung dahin, dass er gegenüber seiner Freundin Gewalt anwendet, für den Zuschauer überraschend. Es ist zwar eine verbreitete Ansicht, dass man den Tätern ihre Verbrechen ansehen könne, aber die Erfahrung widerlegt dies: „Clearly, men who use violence are ordinary people regardless of their educational and social backgrounds, income levels, or ages." (Watanabe 2002: 90-91). Gerade dieser Aspekt, der Kontrast zwischen dem öffentlichen und privaten Auftreten des Täters, wird den Zuschauern der Serie in der Figur des Sōsuke deutlich gemacht.

\section{Die Darstellung der Gewalt in Last Friends}

Die Gewaltszenen in Last Friends werden bis auf die Vergewaltigungsszene vollständig gezeigt, und die Taten werden weder beschönigt, noch werden die Folgen verharmlost. Das einzige Mittel, das verstärkend eingesetzt wird, ist die Filmmusik. So bekommt Sōsuke im Laufe der Serie sein persönliches und sehr bedrohlich wirkendes Musikthema. Durch die angespannte Stimmung, die das Thema vermittelt, erkennt der Zuschauer sofort, dass die betreffende Person nichts Gutes im Schilde führt. Um diesen Kontrast noch zu steigern, wird einige Zeit keine 
Musik eingesetzt, bevor Sōsukes Thema ertönt. Die Stille, in der man jedes Geräusch hört, steigert die Spannung, und die Musik wirkt dadurch wie ein Paukenschlag. Dies steigert die Wirkung um ein Vielfaches und geht dem Zuschauer durch Mark und Bein. Last Friends enthält also ein sehr detailliert ausgearbeitetes Musikkonzept für die Gewaltszenen.

\subsection{Die Entwicklung der Gewalt}

In dem dorama wird der Prozess der Gewaltentwicklung sehr deutlich gezeigt. Zuerst wird dem Zuschauer mit Michiru und Sōsuke ein glückliches Paar vorgestellt. Nachdem die beiden zusammenziehen, enden die Probleme, die am Anfang noch lapidar zu sein scheinen, schließlich aber in einer ersten Gewalthandlung. Sōsuke kontrolliert Michirus Handy, worauf diese ihn verwundert, aber freundlich anspricht. Sōsuke macht seinen Standpunkt klar: Er verdächtigt seine Freundin, ihn mit Ruka zu betrügen. Zur Aufklärung verlangt er von Michiru, die die Vorwürfe zu zerstreuen versucht, konkrete Beweise. Michiru versucht tatsächlich, diese gewünschten Beweise zu besorgen, wird aber noch während sie unterwegs ist von ihm angerufen und aufgefordert, auch ohne Beweise zurück zu kommen. Als sie dem nachkommt, empfängt er sie jedoch mit dem Satz: „Wo ist das Schulalbum [der Beweis]?" Als sie ihm sagt, sie habe es nicht, gerät Sōsuke in Rage. (Folge 1, 38:00).

An dieser Szene, in der zum ersten Mal das Thema der häuslichen Gewalt vorgestellt wird, erkennt man bereits das Muster, das sich durch die weiteren Folgen ziehen wird: Die Unberechenbarkeit des Täters, der seine Meinung sehr schnell ändert, und die Hilflosigkeit des Opfers, das, selbst wenn es versucht die Situation zu entspannen, aus der Sicht des Täters alles falsch macht. Der Zuschauer identifiziert sich mit dem Opfer, das die Reaktionen des Täters nicht nachvollziehen kann. Michiru ist verunsichert, und bei dem Versuch, die Ursache zu finden, ist sie verleitet zu denken, sie selbst sei der Grund. Da der Täter dies bestätigt, projiziert das Opfer die Schuld auf sich und beginnt immer mehr an sich zu zweifeln. 


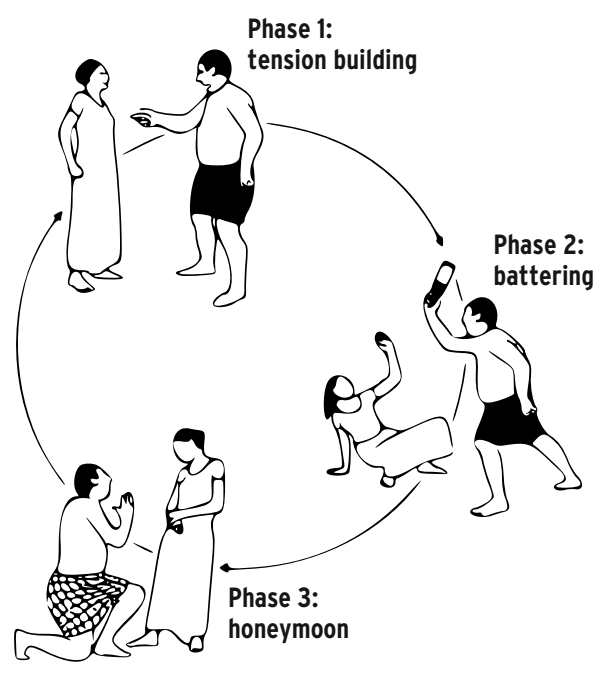

Abbildung 1: Gewaltspirale, Quelle: Coren 2005: 58.

\subsection{Die Gewaltspirale}

An dieser ersten und sehr detailiert dargestellten Gewaltszene ist die Darstellung der Gewaltspirale zu erkennen. Das Konzept der Gewaltspirale wurde 1980 von der amerikanischen Psychologin Dr. Leonore Walker entwickelt (vgl. Coren 2005: 57). Ihr Schema demonstriert, wie die Gewalt in Partnerbeziehungen abläuft. Diese gliedert sich meistens in drei Phasen, die in Abbildung 1 dargestellt sind. Die Phasen werden in dem folgenden Abschnitt kurz erläutert und danach auf die vorher erwähnte Szene bezogen.

\section{Phase 1: Die Aufbauphase}

Das ist die Phase, in der sich die Spannung aufbaut. Es gibt kleinere Streitigkeiten, Anschuldigungen oder geringe körperliche Gewalt, wie Schlagen oder Schubsen. Das Opfer versucht den Täter zu beruhigen und hofft, dass diese Phase vorbei geht (vgl. Coren 2005: 57). In Last Friends findet sich diese Phase der Gewaltspirale in der Szene mit dem Handy. Sōsuke kontrolliert das Handy seiner Partnerin und beschuldigt sie, ihn zu betrügen. Michiru versucht seine An- 
schuldigung zu entkräften und ihn durch einen Beweis zu beruhigen. Als ihr erster Versuch scheitert, baut sich die Spannung so weit auf, dass Sōsuke gegen den Tisch tritt. Erst als die verängstigte Michiru verspricht, einen Beweis zu besorgen, lässt er von ihr ab und beruhigt sich etwas. Als sie jedoch ohne Beweise wiederkommt, auch wenn es auf sein Geheiß war, treten in dieser Situation Aggressionen auf, die sich dann explosionsartig entladen. (Folge 1, 38:00-43:00). Hier findet sich bereits der Übergang zur zweiten Phase.

\section{Phase 2: Die Explosionsphase}

Die zweite Phase ist erreicht, wenn die Spannung in einem Punkt gipfelt, an dem sie in Gewalt umschlägt. Der Auslöser kann beispielsweise ein Wort des Opfers sein, das der Täter als Vorwand dafür nimmt, in Rage zu geraten und die Kontrolle zu verlieren. Der Täter kann dann z. B. Eigentum zerstören und Kindern, Haustieren oder dem Opfer Gewalt antun. Er rechtfertigt sich, indem er sagt, dass er nur seinen Standpunkt klar machen möchte oder dem Partner nur eine Lektion erteilen will. Dennoch dauert der Gewaltausbruch an, bis sich die Spannung gelöst hat, der Täter entkräftet ist oder eine dritte Person (z. B. Familie oder Nachbarn) einschreitet (vgl. Coren 2005: 57). Wenn dies wiederum auf die erste Gewaltszene übertragen wird, erkennt man, dass diese Phase beginnt, als Michiru ohne den „Beweis“ in die Wohnung zurückkehrt. Er packt sie wütend und schubst sie, so dass sie zu Boden fällt. Die Rechtfertigung ist in der Frage „Warum hast du nicht das gemacht, was ich dir gesagt habe?" zu erkennen. Er beginnt ihr Ohrfeigen zu verpassen. Selbst als sie sich schützend auf dem Boden zusammenrollt und ihn anfleht aufzuhören, tritt er immer wieder zu. Erst als er außer Atem ist, hört er auf. Michiru liegt hilflos am Boden. (Folge 1, 43:00-45:00).

Wenn der Täter wieder die Kontrolle erlangt hat und bemerkt, was er getan hat, kommt es zur nächsten Phase, da er das Opfer bzw. die Kontrolle über dieses nicht verlieren möchte.

\section{Phase 3: Die Versöhnung}

Nach der Explosionsphase kommt die Phase der Versöhnung, die auch Phase der Beruhigung genannt wird. Der gewalttätige Partner versucht, seine Reue auszu- 
drücken und es „wieder gut zu machen“. Er entschuldigt sich und nimmt (oft unter Tränen) die Schuld auf sich. Er verspricht sogar, dass so etwas nie wieder passieren werde. Das Opfer wird überschüttet mit Geschenken, Blumen, Schmuck, spezieller Aufmerksamkeit und anderen Zeugnissen der Liebe. Aber diese haben immer die unterschwellige Botschaft des Besitzes und des Anspruchs („Du gehörst mir"). Das Opfer und auch die Personen um sie herum geraten in die Falle, sich bestärkt zu fühlen. Sie hoffen, dass sich das Verhalten des Täters ändert und friedlich wird. Das ist die Gefahr: Die Versöhnungsphase läuft aus, die Spannung baut sich langsam wieder auf und der Kreislauf beginnt von vorne (vgl. Coren 2005: 57-58).

In der besprochenen Szene aus dem terebi dorama scheint sich Sōsuke nach dem Gewaltausbruch wieder zu fangen. Er richtet die verängstigte Michiru auf, entschuldigt sich während er den Tränen nahe ist und drückt sie so fest an sich, dass sie keine Luft bekommt. Michiru löst sich von ihm, entschuldigt sich und sagt, sie suche weiter nach dem Beweis. Dann verlässt sie die Wohnung (Folge 1, 45:00-47:00). Dies ist aber nur ein Teil der Versöhnungsphase. Michiru flüchtet zu Ruka ins Share House ${ }^{4}$.

Sōsuke versucht Michiru auf dem Handy zu erreichen und wartet nach der Arbeit auf sie. Er entschuldigt sich noch einmal und bittet sie, nach Hause zu kommen. Michiru zögert, aber willigt dann doch hoffnungsvoll ein, wieder mit ihm zusammenzuwohnen. (Folge 2, 4:00-7:00). Zusammenfassend sagt die Autorin Mariolein Coren:

The cycle as presented here, can happen hundreds of times in an abusive relationship. Each phase lasts a different amount of time, with the total cycle taking from a few hours to days, weeks, months or more to complete. (Coren 2005: 58)

In der Sequenz aus Last Friends erstreckt sich der komplette Durchlauf der drei Phasen auf zwei Tage. Coren schreibt außerdem, dass man bedenken solle, dass nicht alle Beziehungen, in denen häusliche Gewalt auftritt, in dieses Schema passen. Es könne auch dazu kommen, dass, wenn der Missbrauch weiter geht, die

4 Das Share House ist die Wohngemeinschaft der Freunde. 
Beruhigungs- bzw. Versöhnungsphase wegfällt und das Paar in einer konstant gewalttätigen Beziehung lebt. (Vgl. Coren 2005: 58).

\subsection{Die Gewaltarten}

Die Gewalt, die der Täter gegenüber dem Opfer anwendet, wird in vier Kategorien eingeteilt (vgl. MCADSV 2006: 4-5). Diese Kategorien sind physische, psychische, sozial interaktive und sexuelle Gewalt, das heißt die Kategorien sind nicht auf den körperlichen Aspekt beschränkt. Genau hier lag ein Problem bei der Gesetzgebung in Japan, wie im Kapitel „Die gesetzliche Lage“ noch näher erläutert werden wird.

Die vier Gewaltarten werden auch in dem dorama gezeigt. Um eine Übersicht zu gewährleisten, liste ich sie geordnet nach den Kategorien auf und gebe in Klammern an, in welchen Folgen der Serie sich die entsprechenden Handlungen finden. So lässt sich nachvollziehen, welche Arten nebeneinander auftreten, und es wird sichtbar, ob es eine Steigerung in der Anwendung von Gewalt gibt. Die Auflistung bezieht sich allerdings nur auf die Gewalt, die explizit dargestellt ist. So wird hier z. B. nur die Ohrfeige vom Beginn der Gewaltszene aufgenommen, wenn die weiteren Handlungen nicht zu sehen sind; wenn im nächsten Schnitt eine verprügelte Michiru gezeigt wird, ist nicht erkennbar, welche Unterarten der Gewalt zu diesem Resultat geführt haben und diese können somit auch nicht mit einbezogen werden.

\section{Physische Gewalt}

- Schlagen (Folgen 1, 2, 3, 10)

- Schubsen (Folgen 1,3)

- Schütteln (Folge 1)

- Treten (Folge 1)

\section{Psychische Gewalt}

- Beschuldigungen fremd zu gehen (Folge 1)

- Gewalt an Gegenständen oder mit ihnen werfen (Folgen 1, 5) 
- Überwachung
a) des Handys bzw. über das Handy (Folgen 1, 2, 5)
b) bei der Arbeit (Folgen 2, 3)

- Täter verschafft sich ohne Erlaubnis Zutritt zur Wohnung der Mutter (Folge 2)

- Schuldzuweisungen (Folge 2)

- Aussperren des Opfers aus der gemeinsamen Wohnung (Folge 3)

- Täter verletzt sich selber (Folge 3)

- Täter besticht die Mutter des Opfers mit Geld (Folge 4)

- Drohung sich zu töten (Folge 5)

- Gewalt an Freunden des Opfers
a) Ruka (Folgen 6, 7, 9, 10)
b) Takeru (Folge 9)

- Drohung, den Freunden des Opfers Gewalt anzutun (Folge 10)

\section{Sozial interaktive Gewalt}

- Verbot, Männern die Haare zu schneiden (Folge 2)

- Verbot, Freunde zu treffen (Folge 3)

- Nimmt dem Opfer das Handy weg (Folge 6)

- Verbot zu arbeiten (Folge 6)

\section{Sexuelle Gewalt}

- Sexuelle Nötigung (Folge 5)

- Vergewaltigung (Folge 10)

Die Auflistung zeigt deutlich, dass der größte Teil der Gewalt psychisch ist. Diese Verletzungen sind nicht sichtbar und die Wirkung ist oft stärker. Das Opfer wird so nach und nach entmündigt, von anderen sozialen Kontakten isoliert und wird abhängig vom Täter.

Diese Mittel demonstrieren die Macht und Kontrolle des Täters über das Opfer. Dazu kommt noch die Versöhnungsphase der Gewaltspirale bzw. die Beteuerungen der Liebe und die Zusage, nicht noch einmal Gewalt gegenüber dem Opfer anzuwenden. Das Opfer wird immer unsicherer, unselbstständiger, nimmt 
die Schuld auf sich und hofft, dass sich alles zum Guten wendet. Die Möglichkeit der Unterstützung durch andere Menschen ist eingeschränkt und dadurch ist für das Opfer die Erkenntnis, selbst nicht die Schuld zu tragen, schwer zu erlangen. Selbst wenn sie zu der Erkenntnis gelangt, ist der Gedanke, dass private Sachen nicht in die Öffentlichkeit gehören, im Vordergrund. Das Opfer hat eine Hemmschwelle, die ihm von der Gesellschaft auferlegt wurde. Selbst wenn es diese überwindet, hält sich die Polizei bei privaten Angelegenheiten wegen des $u$ chi-soto ${ }^{5}$-Verhältnisses zurück. Das Opfer ist wie in einem Spinnennetz gefangen und kommt durch eigene Kraft nicht mehr heraus. Darüber hinaus wird deutlich, dass die Vergewaltigung und die Gewalt an den Freunden die Höhepunkte der Gewaltausübung sind. Deshalb werden die Gewalthöhepunkte im kommenden Abschnitt genauer analysiert.

\subsection{Die Gewalthöhepunkte}

In diesem Kapitel werden die Höhepunkte der Gewaltausübung in der Serie mit steigender Intensität wiedergegeben. Die erste Szene ist die, in der Sōsuke Michiru anruft, weil er sie sehen will. Als sie das ablehnt, ruft er ein zweites Mal an und sagt, dass sie nicht mehr vorbeikommen brauche. Er werde sich jetzt töten. (Folge 5, 42:00-45:00). Michiru verlässt deshalb ihre Freunde und will Sōsuke retten. Diese Drohung war allerdings nur ein Trick, um Michiru wieder unter seine Gewalt zu bekommen. Er wartet unverletzt auf sie, nimmt ihr das Handy weg, verbrennt ihr Schulalbum, das sie mit Ruka verbindet, und sagt: „Du gehörst mir! Lass nur uns zwei zusammen sein.“ (Folge 6, 2:42). Danach lebt Michiru wie eine Gefangene. Sōsuke kündigt ihren Job. Sie darf nur mit seiner Erlaubnis das Haus verlassen und bekommt alle halbe Stunde einen Kontrollanruf. Überdies ist sie nun schutzlos den Gewaltattacken von Sōsuke ausgeliefert. Dies erfährt der Zuschauer im Nachhinein, als Takeru, ein Freund, Michiru besuchen kommt, weil dieser sich Sorgen macht. Diese Szene ist sehr wichtig, da dem Zuschauer vor Augen geführt wird, was ohne den unterstützenden Einfluss von Michirus Freunden Ruka und Takeru mit ihr passiert. Selbst wenn der $\mathrm{Zu}-$

5 Uchi (innen, Haus) und soto (draußen, außerhalb) bezeichnet verschiedene Gruppen und definiert ihre Zugehörigkeit. Dieses Verhältnis wird auch durch die Sprache deutlich. 
schauer zuerst denkt, das Ganze sei nicht so schlimm und Michiru könne sich auch allein aus der Situation befreien, wird das mit dieser Sequenz widerlegt. Im Allgemeinen wird dadurch auch deutlich, wie wichtig Freunde und Familie in so einer Situation sind. Auf diesen Aspekt wird später noch genauer eingegangen.

Der zweite Gewalthöhepunkt wird ausgelöst, nachdem Michiru von Takeru befreit wurde. Sōsuke und Michiru begegnen sich zufällig in einem Kaufhaus. Sōsuke möchte, dass sie zurück kommt. Michiru erwidert, dass sie einen neuen Freund habe und nicht mehr zurückkommen werde. Sie läuft weg und Takeru, der zufällig vorbeikommt, begleitet sie zum Share House. Dabei werden sie jedoch von Sōsuke beobachtet (Folge 9, 24:00). Welche Folgen das nach sich zieht, sieht man in der Szene, in der Takeru früh morgens von der Arbeit kommt. Als er sein Fahrrad eine Treppe hinaufträgt, steht Sōsuke am Treppenabsatz. Er schlägt Takeru mit einer Krücke ins Gesicht, so dass dieser rückwärts die Treppe herunterfällt und sich unten noch einmal den Kopf an einem Eisengeländer anschlägt. Er bleibt dort bewegungsunfähig liegen, während Sōsuke die Treppe heruntersteigt. Sōsuke verprügelt Takeru weiter und tritt mit solcher Gewalt auf dessen Hand, dass diese bricht. Drohend sagt er am Schluss, dass er "seiner Michiru“ (Folge 9, 32:00) nicht zu nahe kommen solle (Folge 9, 30:00-32:00). Hier sieht man, welche Folgen Sōsukes Eifersucht haben kann. Es wird zum ersten Mal gezeigt, dass auch die Personen aus dem Umfeld des Opfers in Gefahr sind. In diesem Fall verlässt die Gewalt die Grenze der Paarbeziehung.

Es gibt noch eine weitere Szene in der Sōsuke versucht, über Michirus Freundin Ruka Macht auszuüben. Sōsuke vereinbart telefonisch ein Treffen mit Ruka, woraufhin sie alleine zu ihm in die Wohnung geht. Er droht ihr, ihrer Familie zu schreiben, welche „Krankheit" ${ }^{\text {“6 }}$ sie habe, wovon Ruka sich jedoch nicht einschüchtern lässt. Als er merkt, dass Ruka nicht entsprechend reagiert, ändert er seine Strategie und beschuldigt sie, am Scheitern seiner Beziehung mit Michiru Schuld zu haben. Sie streitet das ab und beschuldigt ihn, Michiru nicht richtig zu lieben. Dies führt dazu, dass Sōsuke Ruka schlägt, die sich daraufhin zwar wehrt, ihm aber kräftemäßig unterlegen ist. Sōsuke geht so weit, ihr die Kleider

${ }^{6}$ Hierbei handelt es sich um Homosexualität bzw. eine Geschlechtsidentitätsstörung. 
vom Leib zu reißen (Folge 9, 40:00): Er erkennt ihre Schwachstelle und nutzt sie aus.

Auch hier wird der rivalisierende Charakter geschädigt. Nicht nur auf der physischen, sondern auch auf der psychischen Ebene. Das zeigt, wie sehr Sōsuke davon überzeugt ist, dass Ruka als Frau und Takeru als Mann mit anderen Männlichkeitsidealen in der Hierarchie unter ihm stehen und er Macht über sie ausüben kann. Wie auch in der Szene mit Ruka beschuldigt er die beiden, Michiru verunsichert und so zu der Trennung bewegt zu haben. Durch seine Handlungen versucht er, diese „Störfaktoren“ zu eliminieren.

Die letzte für das Thema häusliche Gewalt wichtige Szene ist die, in der Michiru ihre Sachen aus der gemeinsamen Wohnung holen soll. Michiru findet bei dieser Gelegenheit einen Gegenstand, der Ruka gehört, und erfährt, dass sie in der Wohnung war. Als Sōsuke erzählt, dass er Rukas Stolz gebrochen habe, schlägt Michiru ihn, woraufhin Sōsuke in Rage gerät und zurückschlägt. Weil Sōsuke damit droht, ihren Freunden etwas anzutun, wehrt sie sich nicht mehr gegen ihn. Er sagt, dass er nicht zulassen werde, dass jemand anderes sie haben kann und vergewaltigt sie (Folge 10, 31:00).

Danach liegt Michiru leblos auf dem Bett und bittet unter Tränen darum, dass Sōsuke ihre Freunde in Frieden lassen solle. Er dürfe dann mit ihr machen was er wolle. Die Tränen machen Sōsuke aggressiv und er verlässt das Zimmer (Folge 10, 38:00). Im Wohnzimmer erkennt er durch Fotos mit ihren Freunden, was er Michiru durch sein Verhalten angetan hat und wie glücklich sie mit den anderen ist. Als Michiru aufwacht, findet sie Sōsukes blutüberströmten Körper und einen Abschiedsbrief, in dem steht, dass er sie frei lasse (Folge 10, 43:00 bis Folge 11, 3:00).

Diese Szene ist der absolute Höhepunkt der häuslichen Gewalt in Last Friends. Die Vergewaltigung ist das Schlimmste, was einer Frau angetan werden kann, aber sie lässt es über sich ergehen, um ihre Freunde zu schützen. Erst als der Täter das Opfer so sieht und erkennt, dass er seine Freundin nicht glücklich macht, sondern im Gegenteil zerstört, fängt er an zu begreifen, was er getan hat. Die Lösung, die die Serie suggeriert, ist der Freitod von Sōsuke. Dieses dramatische Ende mit dem Abschiedsbrief, in dem er erkennt, dass er Michiru so 
lange unterdrücken wird, wie er lebt und er sie durch seinen Tod frei gibt, ist eine Konstruktion der Drehbuchautoren, die neue Perspektiven ermöglichen soll. Diese Wendung gibt der Protagonistin die Möglichkeit, glücklich zu werden und gewährt einen Einblick in die Gefühlswelt des Täters.

Das Ende hat vor allem einen auflösenden Charakter. Es versucht den $\mathrm{Zu}$ schauern die Beweggründe des Täters näher zu bringen. Sōsuke hat seine Partnerin geliebt, aber konnte seine Liebe nur durch den Besitzanspruch demonstrieren. Am Schluss hat er erkannt, dass dies falsch war. So werden die Antipathien gegenüber dem Täter gemindert und der Zuschauer lernt neben der Sicht des Opfers auch die des Täters kennen. In der Serie markiert dies das Ende der Gewalt.

Man erkennt, dass die Intensität der Gewalthöhepunkte chronologisch ansteigt und mit dieser Katastrophe aufgelöst wird. So wurde über einen längeren Zeitraum der Spannungsbogen gehalten und der Zuschauer wurde an die Serie gebunden.

\section{Daten und Fakten zu Domestic Violence in Japan}

Um das Bild, das uns die Serie Last Friends vermittelt, zu bestätigen und zu unterstreichen, werden in diesem Teil der Arbeit die realen Daten und Fakten einbezogen. Als Hintergrund wird im folgenden Abschnitt die gesetzliche Lage zu diesem Thema erläutert und danach wird mit den Ergebnissen einiger Umfragen mit Gewaltopfern verdeutlicht, mit welchen Problemen sich die Opfer konfrontiert sehen.

\subsection{Die gesetzliche Lage}

Im April 2001 wurde nach jahrelangem Kampf einiger Frauengruppen das haigusha kara no bōryoku no bōshi oyobi higaisha no hogo ni kansuru hōritsu (das „Gesetz, das sich sowohl auf die Vorbeugung der Gewalt des Gatten als auch auf den Schutz des Opfers bezieht“ (Ü. d. A.)) verabschiedet. Zum Schutz der Opfer stehen zwei Möglichkeiten zur Wahl.

Entweder kann dem Partner ein sechsmonatiges Verbot auferlegt werden, [...] oder aber der Partner kann aus der gemeinsamen Wohnung ausgewiesen wer- 
den und zwar zunächst für zwei Wochen. Ein Verstoß gegen diese Anordnungen wird strafrechtlich geahndet. (Okaue 2006: 188-89)

Bei Zuwiderhandlung wird eine Gefängnisstrafe von bis zu einem Jahr oder eine Geldstrafe von einer Million Yen (ca. 7.741,50 Euro ${ }^{7}$ ) verhängt.

Allerdings wurde die Gewalt in dem Gesetz nur als körperliche Gewalt verstanden und genauso definiert wie im Strafgesetz. Dennoch müsste das Gesetz auch gegen andere Gewaltformen, wie psychische Gewalt, Schutz bieten (vgl. Okaue 2006: 189). Ein weiteres Problem war, dass das Gesetz nur bei tatsächlich zusammenlebenden Ehepaaren Anwendung fand. Nicht erfasst waren z. B. geschiedene Paare. Deshalb wurde im Jahr 2004 das Gesetz verändert. Viele Kritikpunkte wurden in dem Reformgesetz bedacht und im Mittelpunkt steht das Ziel, dem Opfer zu einem autonomen Leben zu verhelfen, wenn dies vom Opfer erwünscht ist.

Als einer der wichtigsten Reformpunkte ist (1.) die weitergreifende Definition von Gewalt zu nennen. Sie umfasst nun sowohl Taten, die den Körper schädigen, als auch psychische Angriffe. Des Weiteren wird nun auch (2.) die Gewalt eines Ex-Ehemannes geahndet und es kann auch die erwähnte Schutzanordnung gegen ihn erlassen werden. Überdies wurde (3.) die Dauer der Ausweisung aus der Wohnung von zwei Wochen auf zwei Monate verlängert. Somit hat das Opfer mehr Zeit, ein neues Leben zu organisieren. In der neuen Fassung ist auch (4.) ein Zugangsverbot zu den Kindern von sechs Monaten enthalten, da diese als Druckmittel missbraucht werden könnten (vgl. Okaue 2006: 191-92).

Somit ist das Gesetz trotz einiger noch damit verbundener Probleme ein erster Schritt zur Bekämpfung der häuslichen Gewalt. Durch dieses Gesetz wird die Bedeutung der häuslichen Gewalt juristisch anerkannt und der Staat wird verpflichtet, die Gewalt in angemessenem Maße zu verhindern und das Opfer zu schützen. Dadurch sind es nicht mehr nur private Hilfsorganisationen, die den Opfern helfen. Der Staat ist stark eingebunden und muss zum Beispiel auch aufklärende Arbeit an der Allgemeinheit verrichten (vgl. Okaue 2006: 192). Dafür, dass sich das Wissen um das Problem verbreitet, ist das dorama Last Friends ein gutes Beispiel. Die Macher der Serie müssen von einem allgemeinen Interesse

7 Wechselkurs vom 16.3.2009: 1 Euro $=126,601$ Yen. 
der Zuschauer ausgegangen sein, sonst hätte domestic violence nicht eines der Hauptthemen werden können. Das Positive daran ist, dass durch die Serie die Allgemeinheit noch weiter aufgeklärt wird.

Als eine Reaktion auf das Gesetz zitiert der Autor Roger Goodman die Daten von J. Gelb:

As a direct response to the law, arrests in 2002 for spousal abuse were four times higher than in 1998; the National Police and other agencies recorded dramatic increase, up to 50 per cent in reports of domestic abuse. (Gelb 2003: 22, zitiert nach Goodman 2006: 153)

Durch diese Resonanz ist erkennbar, dass das Gesetz den Bedürfnissen der Bevölkerung entspricht und es in diesem Bereich noch viel Bedarf an Aufklärung und Hilfestellungen gibt. Um genau diesen Bedarf genauer zu erläutern, wird im kommenden Abschnitt auf eine landesweite Umfrage eingegangen, die die Probleme der Opfer wiedergibt.

\subsection{Ergebnisse einer Umfrage zu häuslicher Gewalt}

Im Jahr 2004 führte das Kabinettsamt eine landesweite Umfrage zum Thema häusliche Gewalt durch, deren Ergebnisse 2005 veröffentlicht wurden. Im Folgenden werden nun die Ergebnisse vorgestellt und die Probleme analysiert. Abbildung 2 spiegelt die Ergebnisse zu der Gewalterfahrung von Frauen in Japan wider. Es wird deutlich, dass fast jede sechste Frau (15,5\%) schon einmal Gewalt erfahren hat und zwei Drittel dieser Frauen des Öfteren geschlagen wurden. Die beiden Fragen, die in den Abbildungen 3 und 4 wiedergegeben sind, weisen auf einige der Probleme hin, die das Opfer betreffen. Das ist ein wichtiger Punkt für diese Arbeit, da die Antworten in Bezug zu der Darstellung im dorama gesetzt werden können.

Die erste Frage, deren Auswertung Abbildung 3 ist, bezieht sich auf die Personen oder Organisationen, die die Opfer um Rat gefragt haben. Dabei waren Mehrfachnennungen möglich. Auffällig ist, dass fast die Hälfte der Opfer niemandem von der Gewalterfahrung berichtet hat oder berichten konnte. Der Großteil der Menschen, denen sich die Betroffenen anvertrauten, waren Freunde und/oder Familie. Dies ist eine Verbindung, die zu der Serie Last Friends gezo- 


$$
\text { ein paar Mal } \square \text { viele Male }
$$

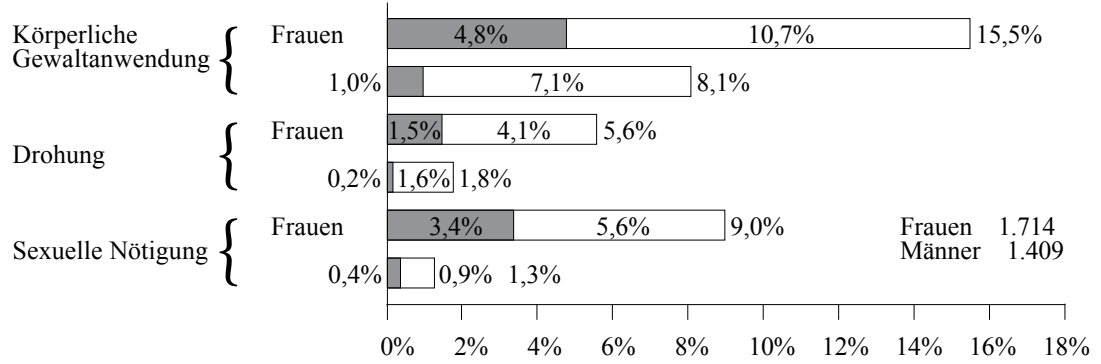

Abbildung 2: Gewalterfahrung in Japan; Quelle: Okaue 2006: 182.

Frage 1: Wen haben Sie bei häuslicher Gewalt zu Rate gezogen?

(Kabinettsamt, Umfrage Okt.-Nov. 2004, veröffentlicht 2005)

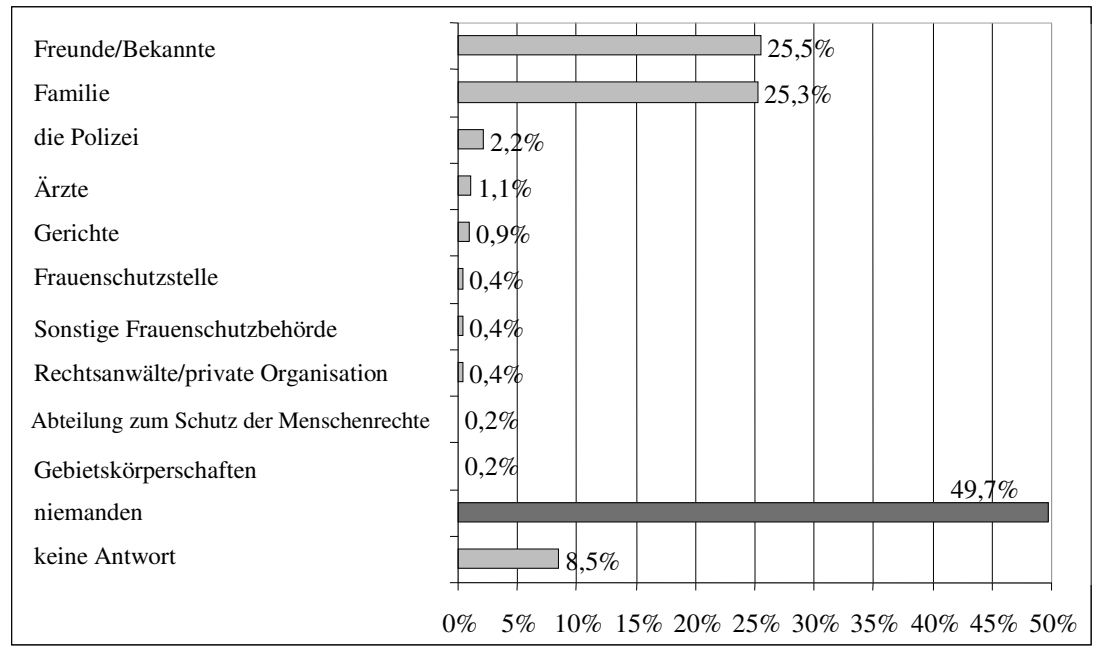

Abbildung 3: Quelle: Okaue 2006: 186 


\section{Fauve Görlach}

Frage 2: Warum haben Sie bei häuslicher Gewalt niemanden zu Rate gezogen?

(Kabinettsamt, Umfrage Okt.-Nov. 2004, veröffentlicht 2005)

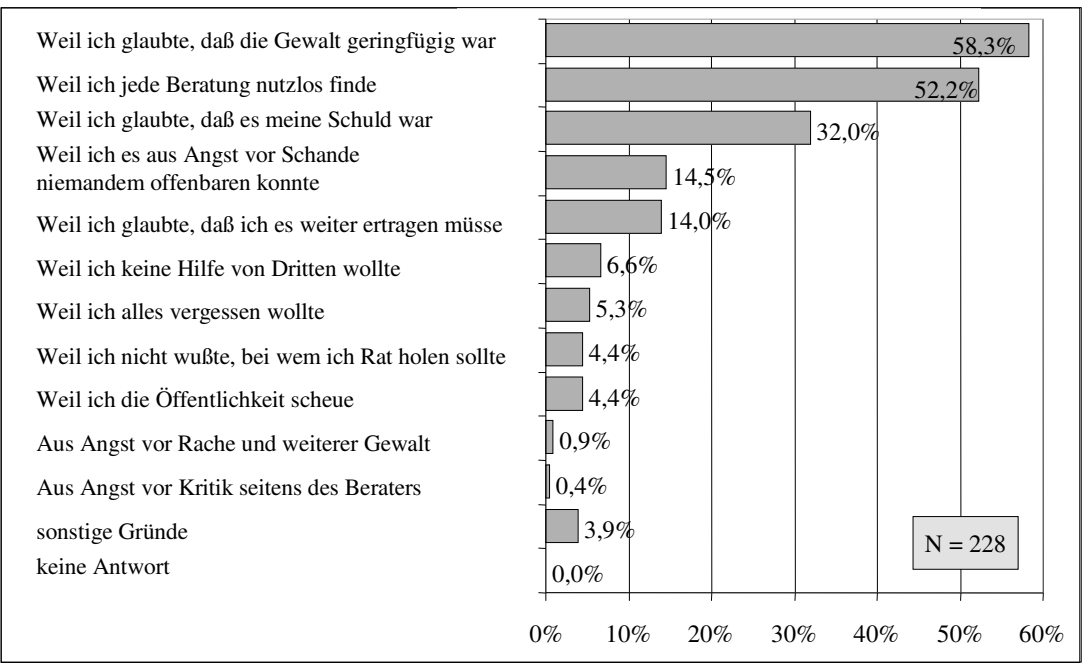

Abbildung 4: Quelle: Okaue 2006: 187

gen werden kann, da die Freunde als das Zentrum der Unterstützung dargestellt werden. Sie beraten Michiru und sind der Gegenpol zu Sōsuke. Dadurch wird die vollkommene Inbesitznahme des Opfers verhindert. Es wurde in der Serie mit der Polizei gedroht, aber diese Drohung wurde nie verwirklich. Der Schritt, eine Person von außerhalb um Hilfe zu bitten, wird offensichtlich vermieden. So erscheint die Polizei in der Umfrage erst auf der dritten Position und nur mit einem geringen Prozentsatz. Die weiteren Hilfsstellen sind erst danach aufgelistet. Es ist wichtig zu betrachten, aus welchen Beweggründen so viele Frauen angegeben haben, niemanden eingeweiht zu haben (vgl. Abbildung 4).

Zirka 58\% der Opfer waren sich nicht der Schwere der Gewalt bewusst und weitere $52 \%$ fanden eine Beratung nutzlos. Das zeigt, wie wichtig es ist, die Bevölkerung über diese Art von Gewalt aufzuklären und zu verdeutlichen, dass eine Beratung etwas verändern kann. Dem Opfer wird geholfen und die Tat wird ernst genommen. Eine weitere Auffälligkeit sind Antworten, wie „Weil ich glaub- 
te, daß es meine Schuld war“ und „Weil ich glaubte, daß ich es weiter ertragen müsse“. Diese Antworten zeugen davon, dass sich das Opfer selbst beschuldigt und das Selbstbewusstsein großen Schaden nimmt. Die Unsicherheit des Opfers vergrößert sich weiter, wenn es niemanden hat, der es unterstützt. Das ist einer der Hauptgründe, warum sich die Opfer aus der Umfrage niemandem anvertraut haben bzw. anvertrauen konnten. Dazu gehören auch die 4,4\%, die nicht wussten, an wen sie sich hätten wenden sollen.

Der nächste Punkt, der mit der Angst vor der Reaktion der Außenwelt zusammenhängt, hat mit der schon erwähnten $u$ chi-soto-Problematik zu tun. Das kann man hier an vier der Antworten erkennen. „Weil ich es aus Angst vor Schande niemandem offenbaren konnte“, „Weil ich keine Hilfe von Dritten wollte“, „Weil ich die Öffentlichkeit scheue“ und „Aus Angst vor Kritik seitens des Beraters“. Das sind zusammen mehr als 25\%. Dieses Denken ist tief verwurzelt in der japanischen Gesellschaft.

Die Scham ist für den Täter ein wichtiger Faktor, da sie verhindert, dass sich das Opfer nach außen wendet. Wenn es dann aus verschiedenen Gründen keine Familie oder Freunde gibt, die das Opfer unterstützen, bleibt das Opfer mit seiner Verzweiflung allein. Einige dieser Punkte wurden auch in dem dorama dargestellt, vor allem der Aspekt, dass sich das Opfer die Schuld gibt. Auch die Scheu, sich an die Polizei zu wenden, geht aus dem dorama hervor. Durch das Einbinden solcher Hintergründe wirkt die Serie gut recherchiert und authentisch.

\section{Fazit}

Das dorama Last Friends gibt einen guten Einblick in die Problematik der häuslichen Gewalt und hat dadurch einen aufklärenden Charakter. Die Gewaltszenen zeigen die Ausmaße, die die Gewalt annehmen kann und beschönigen nichts. Dies und die Andeutung der Hintergründe und Probleme lassen das dorama sehr realitätsnah erscheinen. Dies könnte den Zuschauern ein Bewusstsein für Momente vermitteln, in denen ein Dritter einschreiten muss. Problematisch ist die weiterhin zu beobachtende Existenz von verharmlosenden Gedanken wie dass die Gewalt „ja nur geringfügig“ sei oder dass der Mann das Recht zu solchen 
Taten habe. Ich denke, um eine Diskussion über derartige Probleme auszulösen und viele Haushalte zu erreichen, ist das Medium des Fernsehens sehr gut geeignet. Anhand der Tatsache, dass die Serie viele Auszeichnungen bekommen hat, ist erkennbar, dass die dargestellten Themen gut aufgenommen wurden und eine positive Resonanz bekommen haben. Das domestic-violence-Problem wurde als wichtig genug erachtet, um eines der zentralen Themen des dorama zu werden - Eigentlich sogar in doppelter Weise, da man bei genauerer Betrachtung erkennen kann, dass eine weitere $D V$-Beziehung bei der senpai ${ }^{8}$ von Michiru angedeutet wird.

Rechtlich hat sich, wie diese Arbeit gezeigt hat, für die Betroffenen in Japan einiges getan, wobei besonders die verstärkte Einbindung des Staates eine wichtige Rolle spielt, die einen großen Schritt zur Verbesserung bedeutet. Die Ergebnisse der Umfrage bestätigen, dass die häusliche Gewalt in Last Friends sehr realistisch dargestellt wird. So hat das dorama erfolgreich eine Brücke zwischen der Unterhaltung und der Aufklärung der Zuschauer geschlagen.

\section{Literatur}

Coren, Mariolein (2005): Domestic Violence: a training manual to raise awareness. Phnom Penh: GTZ.

GeLB, J. (2003): „Domestic violence policy in Japan: a comparative perspective“. Paper delivered at the International Sociological Association Meeting on Poverty, Social Welfare and Social Policy. University of Toronto, August 21-24.

Goodman, Roger (2006): „Policing the Japanese family: child abuse, domestic violence and the changing role of the state". In: Rebick, Marcus; Takenaka, Ayumi (Hg.): The changing Japanese family. London; New York: Routledge. S. 147-160.

MCADSV (= the Missouri coalition against domestic and sexual violence) (2006): „A framework for understanding: The nature and dynamics of domestic violence”. http: //www.mocadsv.org/Resources/CMSResources/pdf/dv101.pdf [Stand: 29.3.2009].

Meyer, Dorothe (2009): „Gedanken historischer Persönlichkeiten“. Iwest news Heft 1, S. 23.

Mistutaka, Endo; Moriwaki, Tomonobu; Miyaki, Shogo [Regie] (2008): Last Friends. Pony Canyon (DVD).

OKaue, Masami (2006): „Häusliche Gewalt in Japan“. In: ZJapanR - Zeitschrift für japanisches Recht, 11. Jahrgang, Vol. 11, Nr. 21. S. 181-197.

8 Senpai ist eine ältere und/oder höhergestellte Person. In diesem Fall ist es eine Frau aus dem Friseursalon. 
„Unter der Maske der Liebe“ - Häusliche Gewalt in Last Friends

Watanabe, Noriyoshi (2002): „Japan [domestic violence]”. In: Summers, Randal W.; Hoffman, Allan M. (Hg.): Domestic violence: a global view. Westport, Conn.; London: Greenwood Press. S. 83-95. 
